\title{
Ensemble Learning Based Feature Selection Using Convex Concave Programming
}

\author{
Pinar Karadayi Atas ${ }^{1}$ and Sureyya Akyuz ${ }^{2}$ \\ ${ }^{1}$ Bahçeşehir University Faculty of Engineering and Natural Sciences \\ ${ }^{2}$ Bahcesehir Universitesi Muhendislik ve Doga Bilimleri Fakultesi
}

September 27, 2020

\begin{abstract}
Ensemble feature selection and multiple classifier systems have recently gained importance in machine learning. Ensemble learning improves learning ability by combining several models, an improvement which leads to better predictive performance than a single model. In recent years, ensemble based feature selection approaches have been proposed in which, multiple diverse feature selection methods are combined. These approaches are superior to traditional feature selection techniques in various aspects. In this paper, we propose a novel ensemble based feature selection algorithm using Convex Concave Programming, which is based on ensemble pruning. The optimization model in the pruning step selects the best subset of the ensemble, simultaneously considering the models' accuracy and diversity. The proposed algorithm was tested on multiple data sets and learning performances are compared with various feature selection algorithms. The empirical results shows that the proposed algorithm performs at higher classification accuracy.
\end{abstract}

\section{Hosted file}

$\mathrm{P} \backslash$ selectlanguage $\{$ polish\} $\backslash$ \selectlanguage\{english\}narSureyyaFeatureSelection.pdf available at https://authorea.com/users/362236/articles/483454-ensemble-learning-based-featureselection-using-convex-concave-programming 